\title{
Wissenswertes zur Charakterisierung und Aufbereitung von Rohgrafiten
}

\author{
Wolfgang Lämmerer und Helmut Flachberger
}

Lehrstuhl für Aufbereitung und Veredlung, Department Mineral Resources Engineering, Montanuniversität Leoben, Leoben, Österreich

Eingegangen 8. Juli 2017; angenommen 9. Juli 2017; online publiziert 25. Juli 2017

\begin{abstract}
Zusammenfassung: Die Erzeugung hochwertiger Grafitprodukte aus natürlichen Rohgrafiten setzt ein auf das Rohgut bestmöglich abgestimmtes Aufbereitungsverfahren voraus. Hierbei sind nicht nur die einzelnen Grafittypen zu beachten, sondern auch die für die vielfältigen unterschiedlichen Anwendungsgebiete zu erzielenden Produktspezifikationen, welche einen wesentlichen Einfluss auf die Preisgestaltung - speziell bei Flockengrafiten - haben.

Dieser Beitrag beleuchtet zunächst die Unterscheidung einzelner Grafittypen und behandelt anschließend Zerkleinerungs-, Sortier- und Veredlungsverfahren von Rohgrafiten im Allgemeinen und Flockengrafit im Speziellen. Zusätzlich werden Ausblicke zu Forschungsinhalten mit Rohgrafiten am Lehrstuhl für Aufbereitung und Veredlung der Montanuniversität Leoben gegeben.
\end{abstract}

Schlüsselwörter: Grafit, Charakterisierung von Rohgrafiten, Flotation, Laugung, Spheroidization

Things to Know about Characterization and Beneficiation of Natural Graphites

Abstract: Making high-value products from natural graphite requires fine-tuned beneficiation processes. Both the type of graphite and the specialities in pricing of graphite products need to be considered during flowsheet development. This paper starts with an overview of the characterization of the different types of graphite, followed by the comminution, beneficiation and refining processes of natural graphite in general and with focus on flake graphite. The paper concludes with current research activities at the Chair of Mineral Processing at Montanuniversität Leoben in the field of natural graphite processing.

Dipl.-Ing. W. Lämmerer ( $\square)$

Lehrstuhl für Aufbereitung und Veredlung, Department Mineral Resources Engineering,

Montanuniversität Leoben,

Franz-Josef-Straße 18,

8700 Leoben, Österreich

wolfgang.laemmerer@unileoben.ac.at
Keywords: Graphite, Characterization of graphite, Flotation, Leaching, Spheroidization

\section{Einleitung}

Grafit ist ein vielfältig einsetzbares Industriemineral und daher für die österreichische sowie auch europäische Industrie von enormer Bedeutung. Das Mineral Grafit stellt - wie Diamant - eine allotrope Form von reinem Kohlenstoff dar [1]. Aufgrund seiner besonderen physikalischen und chemischen Eigenschaften und der vielfältigen Anwendungsmöglichkeiten in verschiedensten industriellen Bereichen - vom Bleistift bis zum High-Tech-Endprodukt in Lithium-lonen-Batterien - ist der Bedarf groß. Wegen der Importabhängigkeit von China wurde Grafit daher von der Europäischen Kommission erstmals 2011 auf die Liste der kritischen Rohstoffe gesetzt [2]. Hauptanwendungsgebiete liegen in der Feuerfest-, Gießerei- und Stahlindustrie, darüber hinaus erfolgt ein Einsatz u. a. in Batterien, Farben, Schmierstoffen und Grafitformteilen [3]. Zukünftige Weiterentwicklungen im Bereich der grünen Technologien werden den Grafitmarkt nachhaltig positiv beeinflussen und eine steigende Nachfrage nach natürlichen Grafiten in aufkonzentrierter bzw. veredelter Form ergeben [4]. Derzeit werden jährlich weltweit etwa 1,2 Mio. $t$ an hochwertigen funktionellen Grafitkonzentraten weiterverarbeitet, die aus Grafitlagerstätten gewonnen und aufbereitet werden [5]. Wiewohl Grafite auch auf synthetischem Wege hergestellt werden können, wird eine vollständige Substitution von natürlichen Grafiten durch synthetisch hergestellte Grafite aufgrund des hohen Energie- und somit Kostenaufwandes als sehr unwahrscheinlich erachtet. Der Weiterentwicklung bestehender bzw. der Auffindung neuer Aufbereitungsund Veredlungsverfahren für verschiedene Rohgrafite kommt daher eine besondere Bedeutung zu. Im Zuge des durch die Österreichische Forschungsförderungsgesellschaft (FFG) geförderten und mit Dezember 2015 erfolgreich abgeschlossenen Projektes "Innovative Grafite“ wurden in enger Kooperation mit der Grafitbergbau Kai- 
sersberg $\mathrm{GmbH}$ und den Lehrstühlen für Aufbereitung und Veredlung wie auch für Geologie und Lagerstättenlehre der Montanuniversität Leoben verschiedene Rohgrafite auf Aufbereitbarkeit geprüft, Möglichkeiten der Charakterisierung derselben evaluiert und etablierte wie auch innovative Aufbereitungsverfahren einander vergleichend gegenüber gestellt. Ergänzend wurde an einer Verbesserung der Eigenversorgung durch Prüfung heimischer Vorkommen auf Abbauwürdigkeit gearbeitet.

\section{Unterscheidung von Grafittypen}

\subsection{Allgemeines}

Gleich dem Diamanten besteht Grafit aus reinem Kohlenstoff. Jedoch unterscheiden sich diese durch ihre Kristallstruktur, wodurch sich auch die unterschiedlichen Besonderheiten in ihren physikalischen und chemischen Eigenschaften ergeben. Ein Grafit-Einzelkristall besteht aus mehreren Graphenschichten mit hexagonaler Kristallstruktur, welche durch schwache Van-der-Waals-Bindungen miteinander verbunden sind. Innerhalb der Basalebenen kommen starke kovalente Bindungen zum Tragen. Daraus resultiert eine ausgeprägte Anisotropie, welche sich beispielsweise bei der Härte, der elektrischen und auch der thermischen Leitfähigkeit widerspiegelt [1]. Der Prozess der Grafitisierung - also der Umwandlung von Kohlenstoff mit ungeordnetem Aufbau in eine geordnete grafitische Kristallstruktur - erfolgt unter Metamorphosebedingungen mit erhöhtem Druck und erhöhter Temperatur [6]. Mehrere Methoden wurden beschrieben, um den Grad der Grafitisierung von natürlichen sowie auch synthetischen Grafiten zu bestimmen [7-10]. Die wissenschaftliche Benennung der Stufen kristalliner Ordnung - also mit zunehmendem Grad an Grafitisierung einer kohlenstoffreichen Matrix - erfolgt hierbei nach Kwiecińska et al. [11] über Anthrazit und Meta-Anthrazit als Vorstufen sowie Semi-Grafit und Grafit als Endglieder. In Zusammenarbeit mit dem Lehrstuhl für Geologie und Lagerstättenlehre der Montanuniversität Leoben wurden hierzu Grafite weltweiter Herkunft auf deren physikalische Eigenschaften und deren Kristallinität untersucht und dabei verschiedene Analysemethoden einander vergleichend gegenüber gestellt. Hierbei konnte die Raman-Spektroskopie als geeignete Methode zur Charakterisierung von Grafittypen eruiert werden. Gleichsam als "Nebenprodukt" dieser Forschungsaktivitäten konnte der aus dem Untertagebau der Grafitbergbau Kaisersberg $\mathrm{GmbH}$ stammende mikrokristalline Grafit dem Typus der Semi-Grafite zugordnet werden [9]. Diese Unterscheidung ist nicht nur aus mineralogischer Sicht von Interesse, denn die Stufen der kristallinen Ordnung beeinflussen die Eigenschaften der Grafite wie auch der daraus erzeugten industriellen Produkte in wesentlichem Ausmaß [12-14].

Im wirtschaftlichen Bereich hat sich eine andere Unterteilung durchgesetzt, auf welche im Folgenden näher eingegangen wird und auf deren Struktur die weiteren Inhalte dieses Artikels aufgebaut sind. Die Aufgliederung der natürlichen Grafite erfolgt in wirtschaftlichem Umfeld in mikro- und makrokristalline Grafite, wobei bei makro- kristallinen Grafiten wiederum zwischen Flockengrafiten und Ganggrafiten unterschieden wird. Aus den Unterschieden in den Qualitätsanforderungen an die Konzentrate wie zum Beispiel Grafitgehalt, Kristallitgröße, Korngrößenverteilung, Kornform, elektrische Leitfähigkeit usw. leiten sich zum einen verschiedene Anwendungsgebiete, zum anderen der notwendige Aufbereitungsaufwand ab.

\subsection{Mikrokristalliner Grafit}

Die Bildung von mikrokristallinem Grafit wird auf Kontakt- oder Regionalmetamorphose von Kohlen oder stark kohlenstoffhaltigen Gesteinen zurückgeführt. Vorkommen verzeichnen in der Regel Kohlenstoffgehalte von 50-90\% und können aus mehreren Linsen von wenigen Metern Mächtigkeit, jedoch einigen Kilometern an Erstreckung aufgebaut sein. Etwa $30 \%$ des weltweit abgebauten Grafites sind mikrokristalline Grafite. Der bergmännische Abbau erfolgt meist in selektiver Weise, um marktfähige Qualitäten zwischen 60-90 \% Kohlenstoff ohne zusätzliche Aufkonzentrierung zu erhalten. Lagerstätten für mikrokristallinen Grafit findet man vorrangig in China, Korea, Mexiko und Russland. In Österreich befindet sich in der östlichen Grauwackenzone der Grafitbergbau Kaisersberg, aus dem mikrokristalliner Grafit bergmännisch im Untertagebau hereingewonnen wird. Die Korngröße der Einzelkristalle liegt im Bereich von wenigen Mikron. Dies hat vermutlich auch dazu geführt, dass sich fälschlicherweise die Bezeichnung „amorpher Grafit“ etabliert hat, obwohl alle Grafite eine kristalline Ordnung aufweisen. Mikrokristalliner Grafit ist auf Grund seiner inneren Struktur auch bei größeren Korngrößen von schwarzer Farbe und besitzt keinen Glanz. Die kohlenstoffreiche Matrix ist eng mit der Gangart verwachsen, wodurch sich die Aufbereitung zu qualitativ hochwertigen Grafit-Konzentraten zumeist schwieriger gestaltet als bei makrokristallinen Rohgrafiten [5, 15-18]. Im Zuge des Projektes "Innovative Grafite" konnte mittels einer als umfangreich zu bezeichnenden Explorationstätigkeit im Grafitbergbau Kaisersberg (es wurden 26 Kernbohrungen mit einer Gesamtlänge von mehreren hundert Metern abgeteuft) erfreulicherweise festgestellt werden, dass sich die in Verhieb stehende Lagerstätte in die Teufe fortsetzt und damit gesicherte Lagerstättenreserven von mehreren Zehntausend Tonnen an mikrokristallinem Rohgrafit nachgewiesen werden konnten [19].

\subsection{Makrokristalliner Grafit}

Makrokristalline Grafite werden in Flockengrafite und Ganggrafite eingeteilt. Diese unterscheiden sich durch die Genese, welche sich in den Kohlenstoffgehalten der Rohgrafite und in der Kristallitgröße widerspiegelt.

Flockengrafit lässt sich auf die Metamorphose von kohlenstoffreichem Gestein organischen oder anorganischen Ursprungs zurückführen. Mit über $70 \%$ stellt Flockengrafit den Hauptanteil der weltweit abgebauten Menge an natürlichen Grafiten dar. Ressourcen organischen Ursprungs bilden den Hauptteil an gewinnbaren Lagerstätten [16]. Wie 
im Namen erkennbar, handelt es sich um einzelne Grafitflocken, welche für gewöhnlich isoliert in der umgebenden Matrix eingebettet sind. Kristallitgrößen bis zu $1 \mathrm{~mm}$ sind keine Seltenheit. Die einzelnen Flocken besitzen eine hohe Reinheit und haben einen ausgeprägten Kristallisationsgrad. Typische Lagerstätten sind meist mit einem geringen Kohlenstoffgehalt von 5-30 \% und in massiger Form vorzufinden. Die Gewinnung erfolgt in der Regel über Tage. Durch Einsatz konventioneller Aufbereitungsmethoden werden Konzentratqualitäten mit einem Kohlenstoffgehalt von bis zu $98 \%$ erreicht. Höherwertige Konzentrate mit Kohlenstoffgehalten von bis zu 99,995\% werden durch nachgeschaltete Veredlungsschritte - etwa durch thermischen Aufschluss und/oder Laugung - erzeugt. Flockengrafit ist von silbergrauer Farbe und besitzt einen stark metallischen Glanz. Neben dem Grafitgehalt ist die Flockengröße ein wesentlicher preisbildender Faktor [20]. Dies ist bei der Aufbereitung zu beachten, um die Flocken zu erhalten und damit eine höhere Wertschöpfung zu generieren. Vorkommen von Flockengrafit sind in Österreich von Passau bis ins niederösterreichische Waldviertel zu finden. Gegenwertig erfolgt keine Gewinnung von Flockengrafit in Österreich [19].

Bei Ganggrafit wird angenommen, dass kohlenstoffreiche Fluide in Spalten oder Rissen als Grafit ausgefallen sind. Die subterranen Fluide stammen von Bereichen in großer Teufe, durchdringen organisch oder anorganisch kohlenstoffreiche Schichten und lösen den vorhandenen Kohlenstoff. Unter bestimmten Bedingungen fällt der gelöste Kohlenstoff als hochreiner Grafit in Gängen aus. Die Gewinnung erfolgt unter Tage und in selektiver Abbauweise. Dabei können Grafite mit einem Gehalt von bis zu $99 \%$ Kohlenstoff gewonnen werden. Weltweit sind nur sehr wenige Lagerstätten bekannt und eine aktive Gewinnung von Ganggrafit gibt es zurzeit nur auf Sri Lanka. Ganggrafit wird meist noch in groben Körnungen mittels händischem Klauben sortiert, während bei feinen Körnungen mechanische Aufbereitungsverfahren eingesetzt werden. Ganggrafite besitzen eine hohe Kristallinität und eignen sich somit sehr gut für thermische und elektrische Anwendungen. Von der weltweit abgebauten Menge an natürlichen Grafiten sind etwa $0,4 \%$ den Ganggrafiten zuzuordnen $[5,16]$.

\section{Stand der Technik}

Die Aufbereitung von Rohgrafiten richtet sich nach dem Grafittypus und den geforderten Konzentratqualitäten. Während die Aufbereitungsverfahren bei mikrokristallinen Grafiten und Ganggrafiten häufig aus einer Verschaltung von Zerkleinerungs- und Klassierprozessen bestehen, finden bei Flockengrafiten aufwändiger gestaltete Aufbereitungsverfahren - bestehend aus Zerkleinerung, Sortierung und anschließender Veredlung - Anwendung. Zusätzlich spielt vor allem bei Flockengrafit neben dem Grafitgehalt auch die Flockengröße eine entscheidende Rolle für den erzielbaren Erlös.

\subsection{Zerkleinerung}

An die Zerkleinerung von Rohgrafiten werden unterschiedliche Anforderungen gestellt. Zum einen steht speziell bei Flockengrafit der Aufschluss der einzelnen Grafitflocken als vorbereitender Schritt für nachfolgende Sortierprozesse im Vordergrund, jedoch sollen durch eine schonende Zerkleinerung eben jene Flocken möglichst in der ursprünglichen Größe erhalten bleiben. Diesem Umstand wird durch eine Kombination aus Primärzerkleinerung und einer anschließenden Abfolge an Sortier- und zwischengeschalteten Zerkleinerungsschritten Rechnung getragen. Da Grafit durch seine unpolare Oberfläche an den Basalebenen ein hydrophobes Verhalten zeigt, ist eine Anreicherung mittels Flotation mit geringem Aufwand zu realisieren. Nach der ersten Mahlstufe können frei vorliegende Grafitflocken durch eine Flash-Flotation aus dem Prozesskreislauf ausgetragen werden. Dies dient der Schonung der Grafitflocken, um diese nicht durch Begleitminerale wie Quarz oder andere scharfkantige Körner während der Mahlung zu zerstören. Eine Eigenart von Flockengrafit ist, dass große Flocken zumeist leicht und mit hoher Reinheit aufgeschlossen werden können, wohingegen kleine Grafitkörner meist eng mit der Gangart verwachsen sind, was einer aufwändigen Zerkleinerung bedarf. Durch weitere Aufmahlung des Rückstandes der Rougher-Stufe werden diese noch verwachsenen feinen Grafitflocken aufgeschlossen und der abermaligen flotativen Aufbereitung zugänglich gemacht. Bereits in das Flotationskonzentrat gelangte Grafitflocken werden durch schonende Mahlung und mehrere CleanerStufen von Verunreinigungen befreit. Hierbei ist zu beachten, dass die Begleitminerale lagerstättenbedingt in unterschiedlicher Intensität mit den Grafitflocken verwachsen sein können und somit auch der über Flotation erreichbare maximale Kohlenstoffgehalt lagerstättenbedingten Schwankungen unterliegen kann. Kim et al. [21] haben vier Typen von Verwachsungen definiert, welche mit den Grafitflocken bzw. den Einzelkristalllagen auftreten. Dabei wird zwischen an der Oberfläche anhaftenden Verunreinigungen und Verunreinigungen, welche zwischen den Einzelkristalllagen vorkommen, unterschieden [21]. Jene an der Oberfläche anhaftenden Verunreinigungen können durch Attrition von der Flockenoberfläche gelöst werden, ohne die Flockengröße wesentlich zu beeinflussen. Jene Verunreinigungen zwischen den Lagen können nur durch zusätzliche thermische oder chemische Verfahren und somit kostenintensiv entfernt werden. Neben Backen- und Kegelbrechern für die Primärzerkleinerung finden im Rahmen der Mittelgutmahlung bei der flotativen Aufbereitung von Flockengrafit hauptsächlich Stab- und Kugelmühlen ihren Einsatz. Hierbei werden zur Schonung der Grafitflocken bevorzugt geringere Drehzahlen verwendet, um eine vorwiegend scherende Beanspruchung zu erzielen [22]. Ganggrafite werden zur Aufkonzentrierung aufgrund der selektiven Gewinnung und hohen Reinheit der abgebauten Gänge nur grob zerkleinert und Grobkorn meist durch händisches Klauben sortiert [23].

Zum anderen werden Konzentrate durch gezielte Vermahlung in deren Dispersität und Kornform soweit veredelt, dass die Eigenschaften von Grafit für die nachfolgen- 
Abb. 1: Grafische Darstellung eines möglichen Verfahrensentwurfes für die Aufbereitung von Flockengrafit

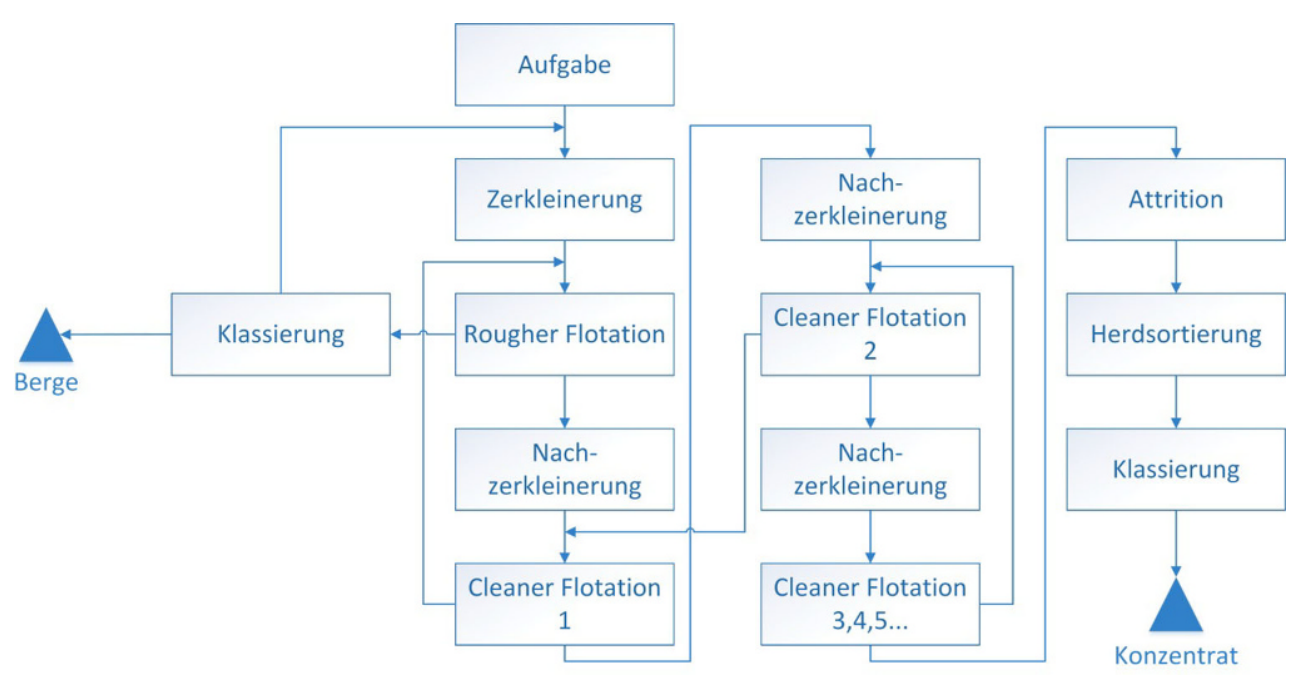

den Anwendungen optimal genutzt werden können. Diese Prozesse werden als "Micronization" oder "Spheroidization“ bezeichnet. Mehr finden Sie hierzu unter Punkt 3.3.3.

\subsection{Sortierung}

Die physikalischen Eigenschaften von Grafit - wie die geringe Dichte und die geringe Oberflächenbenetzbarkeit wurden bereits sehr früh zur Anreicherung von Rohgrafiten ausgenutzt und stellen auch heute noch die Grundlage für die angewandten Sortierprozesse dar. So war die Erfindung der Brüder Bessel im Jahr 1877 - Besitzer des Grafitbergbaues Kropfmühl in Passau - der Grundstein für die Entwicklungen im Bereich der Flotation. Durch Zugabe von Kohlenwasserstoffen zur Verstärkung des Sammlereffektes und der Erzeugung von aufsteigenden Gasblasen durch Kochen der Suspension erreichten sie als Erste eine Trennung von Grafit und Gangart durch Flotation und patentierten dieses Verfahren [24]. Zuvor wurde das gewonnene Rohgut meist über Dichtesortierung angereichert.

Die Flotation ist weltweit das Standardverfahren zur Aufkonzentrierung von Rohgrafiten. Aufgrund der geringen Oberflächenbenetzbarkeit ist Grafit ein natürlich hydrophobes Mineral und benötigt somit nur eine verstärkende Wirkung durch Einsatz von Sammlern. Das Reagenzienregime zur Grafitflotation besteht meist aus Schäumern wie Methylisobuthylcarbinol (MIBC) oder Pine oil, des Weiteren aus Kohlenwasserstoffen wie Petroleum, Diesel oder Schweröl als Sammler und - in Abhängigkeit von der Gangart - aus Standarddrückern wie Wasserglas, Milchsäure oder Ligninsulfonat. Zur Einstellung des pH-Wertes werden meist Kalk oder Soda verwendet [22, 23, 25, 26]. In mehreren Arbeiten wurde über die Auswahl von geeigneten Schäumern zur Flotation von Grafit berichtet. Es stellte sich vor allem MIBC oder MIBC als Mischung mit Ethanol als geeignetes Flotationsreagenz heraus [27, 28]. Der Flotationsprozess setzt sich aus einer Rougher-Flotation mit mehreren Cleaner-Stufen zusammen. Um den Rohgrafit von einem Aufgabegehalt von 10-30\% Kohlenstoff auf ein Flotationskonzentrat mit bis zu $98 \%$ Kohlenstoff anzureichern, werden bis zu fünf Cleaner-Stufen und mehr durchgeführt. Zwischengeschaltet sind Mahl- und Klassierprozesse, um die Aufschlussverhältnisse zu verbessern, jedoch ein Übermahlen der Grafitflocken zu verhindern. Durch Attrition werden oberflächlich anhaftende Verunreinigungen an den Grafitflocken der Flotationskonzentrate abgelöst und über die letzten Cleaner-Stufen abgereichert. Da speziell kleinere Grafitpartikel fein verwachsen sind und somit eine intensive Mahlung für einen ausreichenden Aufschluss nötig ist, kann dies zu einem oberflächlichen Verschmieren der Gangart führen. Dies bewirkt, dass diese mit Grafit überzogene Gangart fälschlicherweise in das Flotationskonzentrat ausgetragen wird. Um diesem Umstand Rechnung zu tragen, wird in manchen Aufbereitungsanlagen eine zusätzliche Nachreinigung der Flotationskonzentrate mit Stoßherden durchgeführt $[23,29]$. Die an den Grafiten anhaftenden Reagenzien werden durch intensive Trocknung - zum Beispiel bei $350^{\circ} \mathrm{C}$ - wieder vom Flotationskonzentrat entfernt, um nicht die nachfolgende Produktverarbeitung negativ zu beeinflussen. Anschließend wird das getrocknete Flotationskonzentrat je nach Kundenspezifikation klassiert und für den Versand abgepackt oder in weiteren Prozessschritten veredelt. Ein möglicher Verfahrensentwurf zur Aufbereitung von Flockengrafit ist in Abb. 1 dargestellt.

\subsection{Veredlung}

Hochtechnologische Anwendungen erfordern Qualitäten mit sehr hohen Kohlenstoffgehalten. Wie unter Punkt 3.1 bereits erörtert, lassen sich Rohgrafite mit physikalischen bzw. physikalisch-chemischen Methoden nicht beliebig hoch aufkonzentrieren, da Verunreinigungen auch zwischen den einzelnen Schichten der Grafitflocken liegen. Für nukleartechnische Anwendungen oder bei Lithium-IonenBatterien werden jedoch Reinheiten größer $99 \%$ Kohlenstoff benötigt. Um dies zu erreichen, werden thermische und/oder chemische Verfahren eingesetzt und Konzentrate mit bis zu 99,995\% Kohlenstoff erreicht. Die Modifikation der Morphologie gehört ebenfalls zu den Veredlungs- 
schritten bei Grafitkonzentraten. Durch gezielte Mahlung kann die Partikelform der Grafite verändert werden, um den geforderten Eigenschaften als "High-End“-Produkt in der Anwendung zu genügen. "Spheroidization", also das Runden von Grafitflocken, soll hier beispielhaft behandelt werden.

\subsubsection{Chemische Verfahren}

Die chemische Reinigung von Flotationskonzentraten stellt das Standardverfahren zur Herstellung von hochreinen Grafiten dar. Aufgrund der chemischen Stabilität von Grafit können Verunreinigungen entweder im sauren - als Säurelaugung bezeichnet - oder im alkalischen Milieu als warmer Sodaaufschluss bezeichnet - gelaugt werden, ohne grafitische Bestandteile wesentlich anzugreifen. Im Konzentrat enthaltene Verunreinigungen können jedoch einen nicht unbedeutenden katalytischen Effekt hervorrufen und somit auch zu Grafitverlusten während der Laugung führen $[1,16,30]$. Arbeiten zu unterschiedlichen Laugungsverfahren wurden bereits von mehreren Autoren verfasst und deren Effizienz bei unterschiedlichen Rohgrafiten beurteilt. In Abstimmung auf die zu laugenden Verunreinigungen werden verschiedene Säuren wie Salzsäure $(\mathrm{HCl})$, Flusssäure $(\mathrm{HF})$, Schwefelsäure $\left(\mathrm{H}_{2} \mathrm{SO}_{4}\right)$ oder Salpetersäure $\left(\mathrm{HNO}_{3}\right)$ in Reinform oder als Mischungen eingesetzt. Die Säurelaugung ist eine sehr effektive Methode, um Verunreinigungen zu entfernen. HF ist vor allem bei Silikaten gegenüber $\mathrm{HCl}$ und $\mathrm{H}_{2} \mathrm{SO}_{4}$ im Vorteil, wohingegen HF allfällig enthaltenen Pyrit nicht zu lösen vermag. Die Säurelaugung stellt vor allem im chinesischen Raum das Standardverfahren dar. Der warme Sodaaufschluss erfolgt als Abfolge aus Röstung mit Natriumhydroxid $(\mathrm{NaOH})$, Laugung mit Wasser und anschließender Säurelaugung mit $\mathrm{HCl}$ oder $\mathrm{H}_{2} \mathrm{SO}_{4}$ [31-35]. Am Lehrstuhl für Aufbereitung und Veredlung der Montanuniversität Leoben wurden verschiedene Laugungsvarianten auf deren Eignung zur Laugung von Flockengrafit getestet und die Auswirkungen auf die Grafitstruktur, die Dichte und die BET-Oberfläche erhoben. Im Zuge der Versuche konnten mittels des warmen Sodaaufschlusses und mittels des Einsatzes von HF Konzentrate mit einer Reinheit von bis zu 99,98\% Kohlenstoff erzeugt werden. Die Laugungsvarianten hatten keinen wesentlichen Einfluss auf die physikalischen Eigenschaften wie Oberfläche, Dichte und Kristallinität der gewonnenen Endprodukte [36]. Diese Ergebnisse werden durch eine ähnlich angelegte Studie von Xie et al. [37] bei mikrokristallinem Grafit bestätigt.

\subsubsection{Thermische Verfahren}

Bei thermischen Verfahren werden Flotationskonzentrate oder bereits chemisch gelaugte Konzentrate nochmals in inerter Atmosphäre auf Temperaturen von bis zu $3000{ }^{\circ} \mathrm{C} \mathrm{er}$ hitzt. Verunreinigungen treten dabei in die Gasphase über und können abgezogen werden. Dieser Prozess erfordert hohe Investitions- und Betriebskosten. Produkte mit Kohlenstoffgehalten von bis zu 99,995\% können erzeugt werden, welche in Spezialanwendungen wie Luft- und Raumfahrt ihren Absatz finden [38].

\subsection{3 "Spheroidization"}

Zusätzlich zum Reinheitsgrad spielt bei vielen Hochtechnologieanwendungen auch die Kornform eine wesentliche Rolle. Durch die Anisotropie von Grafit ergeben sich zwei Flächen mit unterschiedlichen Eigenschaften. Entlang der Basalebene stellt Grafit einen sehr guten thermischen sowie auch elektrischen Leiter dar, während quer zur Basalebene - also zwischen den einzelnen Ebenen - Grafit als thermischer sowie auch elektrischer Isolator angesehen werden kann. Aufgrund der großen Unterschiede der Oberflächenenergien finden kaum chemische Reaktionen an den Basalebenen statt, während sich Grafit an den Kanten als reaktionsfreudiger darstellt [1]. Dieser Umstand führt dazu, dass - je nach Anwendung - verschiedene Kornformen und -größen gefragt sind. Am Beispiel der Lithiumlonen-Batterien - wo Grafit als Anode eingesetzt wird kann der Einfluss entsprechender Veredlungsverfahren dargestellt werden. Die Lithium-Ionen-Interkalation - also die Einlagerung der Lithium-Ionen zwischen den einzelnen Basalebenen - findet während der Ladephase einer Batterie nur an den Kanten oder an Defekten einer Grafitflocke statt. Quer zur Basalebene ist dies nicht möglich (siehe dazu Abb. 2a). Durch gezielte Vermahlung mit Mühlen zur Feinstmahlung werden Grafitflocken in ihrer Form so verändert, dass die Grafitfocken eine runde Form durch Einrollen derselbigen erhalten. Dies hat zum einen zur Folge, dass sich die für die Interkalation mit den Lithium-Ionen verfügbaren Kantenoberflächen erhöhen (siehe dazu Abb. 2b). Zum anderen erhöht sich die erreichbare Packungsdichte bei der Anodenherstellung und somit wird Kapazität der Batterie erhöht. Diese als „Potato-Shaped Graphite“ bezeichneten Produkte werden im Anschluss noch durch Laugung gereinigt und die Oberfläche mit leitfähigem Kohlenstoff überzogen. Durch diese Veredlungsschritte werden Ladeund Entladezeiten sowie auch die Kapazität der Batterien positiv beeinflusst [39-43].

Abb. 2: Schematische Darstellung der verbesserten $\mathrm{Li}$ thium-Ionen-Interkalation bei Grafitflocken (a) und bei „Potato-Shaped Graphite" (b) nach $[40,41]$

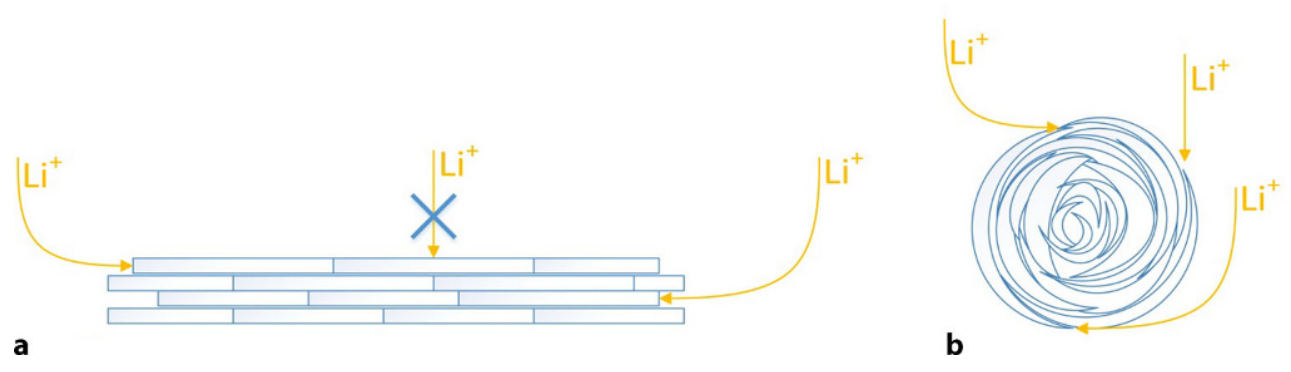




\section{Innovative Aufbereitungsmethoden}

Innovative Aufbereitungsmethoden standen auch im Fokus des FFG Projektes "Innovative Grafite.. Hierzu wurden alternative Aufbereitungsmethoden im Bereich der Zerkleinerung sowie auch der Sortierung näher betrachtet.

\subsection{Elektrodynamische Fragmentierung}

Die elektrodynamische Fragmentierung gilt als vielversprechende Zerkleinerungsmethode für den primären sowie auch sekundären Rohstoffsektor. Über Entladungen von Hochspannungsimpulsen wird das zu zerkleinernde Gut beansprucht und bevorzugt an den Mineralphasengrenzen zerkleinert. Dies verspricht einen frühen und damit möglichst groben Aufschluss der beteiligten Mineralphasen. Die elektrodynamische Fragmentierung basiert auf der Entladung hochenergetischer Impulse zwischen zwei Elektroden. Dielektrische Feststoffe - also elektrisch schwach oder nicht leitende, nicht metallische Substanzen - welche sich zwischen den Entladungspunkten befinden, werden von den Impulsentladungen durchschlagen, sofern die Pulsanstiegsdauer unterhalb einer kritischen Zeitspanne liegt. Diese kritische Zeitspanne ist abhängig von der Durchschlagsfestigkeit des den Feststoff umgebenden Mediums, da die Entladung nicht durch das Medium, sondern durch den Feststoff erfolgen soll. Bei der Verwendung von Wasser liegt die kritische Pulsanstiegsdauer bei ca. $500 \mathrm{~ns}$ [44]. Auf Grund von Unterschieden in den dielektrischen Eigenschaften verschiedener Phasen innerhalb des Festkörpers verläuft die Impulsentladung vorzugweise entlang von Phasengrenzen. Beim Anlegen einer Spannung zwischen zwei Elektroden entsteht zunächst ein inhomogenes elektrisches Feld, wodurch die Oberflächen der einzelnen Phasen im Festkörper unterschiedlich stark polarisiert werden und sich sogenannte Streamer ausbilden. Entlang dieser Streamer bildet sich ein Plasmakanal, dessen plötzliche Ausdehnung eine Druckwelle ergibt, welche sich durch den Feststoff fortsetzt. An akustischen Diskontinuitäten wird diese Druckwelle reflektiert, wodurch Zugspannungen in den Festkörper eingebracht werden und diese die Gesteinsfestigkeit überschreiten. Dies führt zum Versagen und somit zur Zerkleinerung des Festkörpers. Da sich zum einen der Plasmakanal überwiegend an den Oberflächen der Phasen ausbildet und zum anderen die Reflexion der Druckwelle an den Phasengrenzen stattfindet, ist von einer intergranularen Zerkleinerung auszugehen [45].

Im Zuge der Projektarbeit wurden vergleichende Zerkleinerungsuntersuchungen mit konventionellen Zerkleinerungsaggregaten und mit elektrodynamischer Fragmentierung bei der Selfrag AG im Labormaßstab an einem Rohgrafit aus Mosambik durchgeführt. Für beide Methoden wurde der spezifische Nettoenergieeintrag zur Zerkleinerung innerhalb einer definierten Dispersität erhoben und das Aufschlussverhalten in Bezug auf Flockengrafit für beide Prozesse beurteilt.

Um den erforderlichen spezifischen Nettoenergieeintrag für die Zerkleinerung eines Rohgrafites von einer Aufgabekorngröße $<40 \mathrm{~mm}$ auf eine Produktkorngröße $<1 \mathrm{~mm}$ zu ermitteln, erfolgte die Versuchsdurchführung jeweils nach der von Steiner entwickelten „Optimierten Zerkleinerungskette" [46]. Anschließend wurden beide Zerkleinerungsprodukte $<1 \mathrm{~mm}$ mittels einer Schwimm-/ Sink-Analyse in Dichteklassen aufgeteilt und damit die Aufschlussgrade von Grafit innerhalb einzelner Korngrößenklassen bestimmt. Diese Merkmalsklassenanalyse brachte zwei interessante Erkenntnisse zutage: Zum einen ist der spezifische Nettoenergieeintrag bei der elektrodynamischen Fragmentierung im Vergleich zur konventionellen Zerkleinerung beim untersuchten Dispersitätssprung wesentlich höher, zum anderen ist der Aufschluss bei der elektrodynamischen Fragmentierung bereits in gröberen Korngrößenbereichen besser.
Abb. 3: Grafische Darstellung des Anteils aufgeschlossener Grafitflocken in Abhängigkeit unterschiedlicher Korngrößenklassen von elektrodynamischer Fragmentierung bzw. konventioneller Zerkleinerung

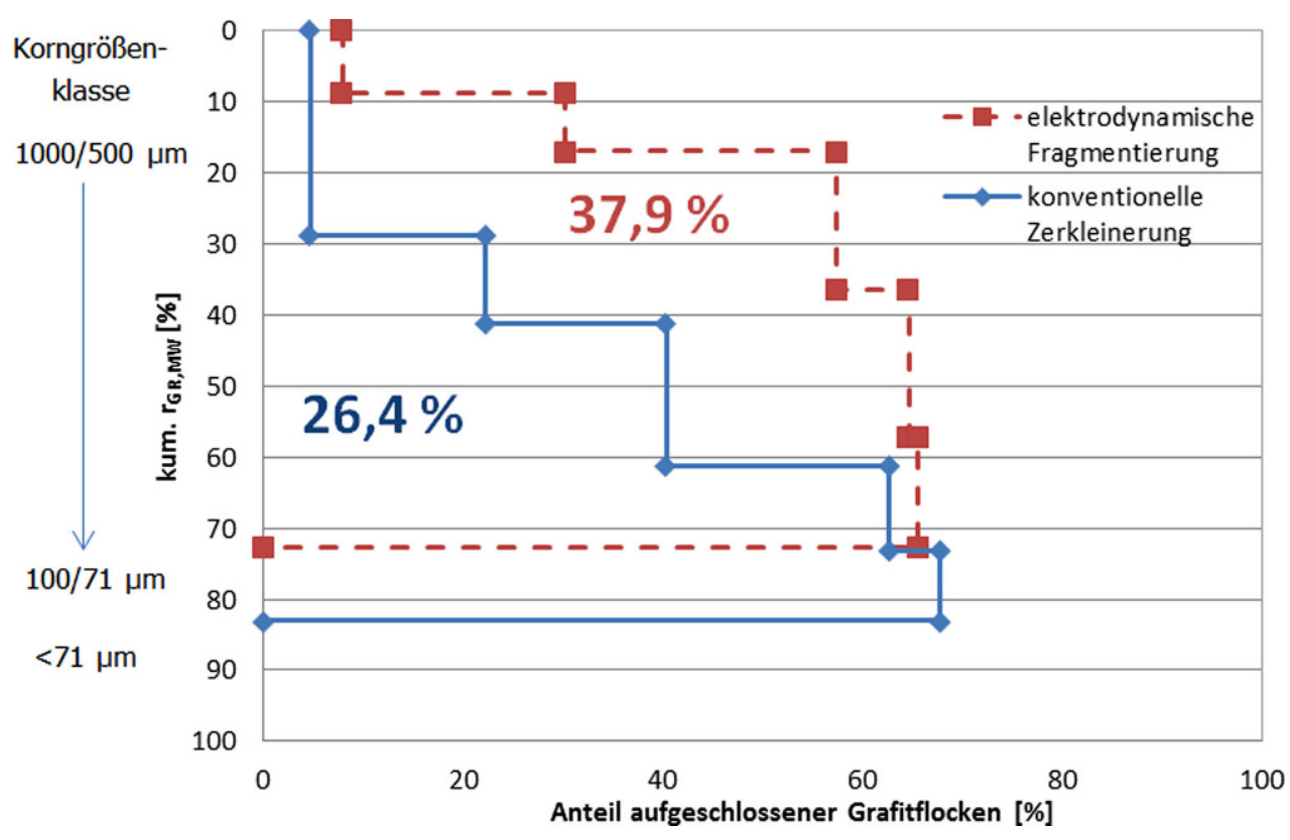


Zur Veranschaulichung ist in Abb. 3 eines der Ergebnisse aus den durchgeführten Untersuchungsreihen beispielhaft dargestellt. Auf der Ordinate ist das Inhaltsausbringen an Grafit in den untersuchten Korngrößenklassen aufgetragen, auf der Abszisse der aufgeschlossene Anteil an Grafit. Die dabei entstehenden Flächen - strichliert umrandet für die elektrodynamische Fragmentierung und ungebrochen umrandet für die konventionelle Zerkleinerung - stelIen die Inhalte an aufgeschlossenen Grafitflocken im Korngrößenbereich von $1 \mathrm{~mm}$ bis $71 \mu \mathrm{m}$ dar. Außerhalb der Umrandungen sind verwachsene Grafitflocken bzw. Feingut $<71 \mu \mathrm{m}$ enthalten. Durch Aufsummieren der Inhalte an aufgeschlossen vorliegenden Grafitflocken der untersuchten Korngrößenklassen zeigt sich, dass bei der konventionellen Zerkleinerung 26,4\% des Grafitinhaltes in aufgeschlossener Form vorliegen, während sich dieser Anteil bei der elektrodynamischen Fragmentierung deutlich auf 37,9\% erhöht.

Die Kombination von elektrodynamischer Fragmentierung - mit den Zielen einer frühzeitigen Freilegung der Grafitflocken bzw. Verbesserung der Aufschlussverhältnisse und anschließender konventioneller Zerkleinerung - mit dem Ziel einer Erzeugung der geforderten Dispersitäten kann somit eine interessante Alternative darstellen.

\subsection{Triboelektrische Bandscheidung}

Die Sortierung von Mineralgemengen in elektrostatischen Feldern wird in der Industrie für mineralische sowie auch sekundäre Rohstoffe eingesetzt. Die Elektroscheidung ist gleich der Flotation - ein grenzflächengesteuertes Sortierverfahren, kommt jedoch weitestgehend ohne den Einsatz von Reagenzien aus und wird in trockener Weise betrieben. Zur Sortierung werden Unterschiede in der Oberflächenleitfähigkeit oder den Oberflächenladungen der Körner ausgenutzt. Diese Ladungsunterschiede müssen jedoch zuvor auf den Kornoberflächen erzeugt werden. Der Effekt der triboelektrischen Aufladung wird bei der Nichtleiter-/ Nichtleiter-Trennung ausgenutzt. Durch gegenseitiges Reiben der Körner unterschiedlicher Mineralphasen oder durch Reiben der Körner an Bauteiloberflächen der Aggregate werden Ladungen verschiedener Polarität oder Größe durch Unterschiede in den Dielektrizitätskonstanten bzw. den Elektronenaustrittsenergien an den Kornoberflächen erhalten. Dieser Vorgang kann durch klimatische Bedingungen wie Luftfeuchte oder Temperatur oder auch durch die Konditionierung des Rohgutes - Rohgutfeuchte, Rohguttemperatur, oberflächenaktive Reagenzien usw. maßgeblich beeinflusst werden. Die Methoden der Kontaktpolarisation oder der Koronaaufladung werden speziell bei der Leiter-/Nichtleiter-Trennung eingesetzt, um anhand der elektrischen Leitfähigkeit der Mineralphasen unterschiedliche Ladungen an den Oberflächen zu generieren [47].

Die Trennung der solcherart vorbereiteten Körner erfolgt in einem elektrostatischen Feld mittels des Einsatzes von Walzenscheidern oder Freifallscheidern und war bisher auf Korngrößen im Dispersitätsbereich 2/0,1 mm begrenzt. Durch die Entwicklung des triboelektrostatischen
Bandscheiders der Firma ST Equipment and Technologies LLC (STET) kann nunmehr auch der Korngrößenbereich $<0,1 \mathrm{~mm}$ mittels Elektroscheidung aufbereitet werden, der bislang ausschließlich der Flotation vorbehalten war.

Durch Versuche im Pilotmaßstab mit einem Rohgrafit aus Tansania konnte gezeigt werden, dass dieser Bandscheider für die Aufbereitung des untersuchten Flockengrafites geeignet ist. So konnten im einstufigen, reagenzienfreien Betrieb Vorkonzentrate mit einem Masseausbringen von etwa $50 \%$ und einem Inhaltsausbringen an Grafit von bis zu $94 \%$ erzeugt werden. Bei weiteren Versuchen mit anderen Rohgrafiten konnten Konzentrate in mit Rougherkonzentraten vergleichbaren Qualitäten erzeugt werden. Somit hat dieser Bandscheider nach derzeitigem Untersuchungsstand das Potenzial, eine Alternative in trockener Betriebsweise zur derzeit dominierenden Flotation zur Aufbereitung qualitativ hochwertiger Grafitprodukte darzustellen. Auch eine Kombination beider Prozesse ist denkbar, die Elektroscheidung zur Bergevorabscheidung und die Flotation zur Weiterverarbeitung der Vorkonzentrate zu den vielfältigen funktionellen Füllstoffprodukten [48, 49].

Die diesbezüglichen experimentellen Arbeiten und die Darstellung detaillierter Ergebnisse zu den eben genannten Forschungsaktivitäten - der elektrodynamischen Fragmentierung und der triboelektrischen Bandscheidung - werden in einer der nächsten BHM-Veröffentlichungen präsentiert.

\section{Zusammenfassung}

Die Nutzung natürlicher Grafite in industriellen Anwendungen und vor allem in grünen Technologien führt zu einer stetigen Steigerung der Verbrauchsmengen. Neben der Exploration neuer Ressourcen werden bereits in Verhieb stehende Lagerstätten weiter genutzt, auch wenn diese geringere Grafitgehalte oder komplexere Verwachsungsverhältnisse aufweisen. Durch die optimale Gestaltung der Aufbereitungsverfahren können selbst geringhaltige Lagerstätten genutzt und die hohen Anforderungen der Industrie an die erzeugten Produkte erfüllt werden.

Die Zerkleinerung von Rohgrafiten spielt eine wesentliche Rolle als Vorbereitung für die zum Einsatz gelangenden Sortierverfahren. Durch eine schonende Zerkleinerung können Grafitflocken vorsichtig freigelegt werden, ohne diese zu zerstören. Bei fein verwachsenen Rohgrafiten gilt es diese aufzuschließen, ohne die Gangart mit Grafit zu überziehen und so eine Trennung mittels Flotation negativ zu beeinflussen. Oberflächliche Verunreinigungen sind bei Grafitflocken über eine schonende Attrition und mehrere Reinigungsstufen zu reduzieren.

Die Flotation ist als Standardverfahren bei der Grafitaufbereitung sehr gut beschrieben und etabliert. Als Reagenzien werden MIBC als Schäumer, Petroleum als Sammler und Wasserglas als Drücker verwendet.

Hochreine Grafitprodukte können nicht über physikalische, sondern ausschließlich über chemische oder thermische Verfahren erzeugt werden. Zur Säurelaugung werden $\mathrm{HCl}, \mathrm{HF}, \mathrm{H}_{2} \mathrm{SO}_{4}$ oder Mischungen davon herangezogen. Alternativ zu HF kann über einen warmen Sodaaufschluss mit 
anschließender Säurelaugung ein Grafitkonzentrat von bis zu 99,99\% Kohlenstoff erzeugt werden.

Über abschließende Veredlungsschritte, etwa durch "Spheroidization", werden dem Rohstoff "Grafit" besondere funktionelle Eigenschaften verliehen, welche ihn in der modernen Technik unabdingbar machen und die Wertschöpfung für den Produktionsbetrieb wesentlich erhöhen.

Innovative Aufbereitungsmethoden wie etwa die elektrodynamische Fragmentierung oder die triboelektrostatische Bandscheidung können eine Alternative zu konventionellen Methoden darstellen. Zum einen kann ein frühzeitiger Aufschluss von Grafitflocken die Wertschöpfung der Produkte erhöhen, zum anderen können durch die trockene Aufbereitungsmethode Ressourcen in Gebieten mit begrenztem Zugang zu Prozesswasser erschlossen werden.

Acknowledgements. Open access funding provided by Montanuniversität Leoben.

Open Access Dieser Artikel wird unter der Creative Commons Namensnennung 4.0 International Lizenz (http://creativecommons.org/licenses/ by/4.0/deed.de) veröffentlicht, welche die Nutzung, Vervielfältigung, Bearbeitung, Verbreitung und Wiedergabe in jeglichem Medium und For mat erlaubt, sofern Sie den/die ursprünglichen Autor(en) und die Quelle ordnungsgemäß nennen, einen Link zur Creative Commons Lizenz beifügen und angeben, ob Änderungen vorgenommen wurden.

\section{Literatur}

1. Pierson, H. O.: Handbook of Carbon, Graphite, Diamond, and Fullerenes, New Jersey, U.S.A.: Noyes Publications, 1993

2. Europäische Kommission: Mitteilung der Kommission: Grundstoffmärkte der Rohstoffe: Herausforderungen und Lösungsansätze, 2011

3. Donald, W. O.: Mineral Commodity Summaries, USGS National Minerals Information Center: Graphite (natural), https://minerals. usgs.gov/minerals/pubs/commodity/graphite/mcs-2017-graph.pdf (13.04.2017)

4. Frey, C.: Verwendung von Naturgraphit in Lithium-lonen-Akkus Auslöser eines Explorationsbooms, AKK - Frühjahrstagung 2016 Meitingen, Deutschland, 2016

5. Scopings, A.: What makes graphite projects tick?, $6^{\text {th }}$ Graphite \& Graphene Conference, Berlin, 2017

6. Beyssac, O.; Rouzaud, J.-N.; Goffé, B.; Brunet, F; Chopin, C.: Graphitization in a high-pressure, low-temperature metamorphic gradient: A Raman microspectroscopy and HRTEM study, Contributions to Mineralogy and Petrology 143 (2002), Nr. 1, pp 19-31

7. Kwiecinska, B.; Suárez-Ruiz, I.; Paluszkiewicz, C.; Rodriques, S.: Raman spectroscopy of selected carbonaceous samples, International Journal of Coal Geology 84 (2010), 3-4, pp 206-212

8. Rodrigues, S.; Marques, M.; Suárez-Ruiz, I.; Camean, I.; Flores, D. Kwiecinska, B.: Microstructural investigations of natural and synthetic graphites and semi-graphites, International Journal of Coal Geology 111 (2013), pp 67-79

9. Rantitsch, G.; Lämmerer, W.; Fisslthaler, E.; Mitsche, S.; Kaltenböck $\mathrm{H}$.: On the discrimination of semi-graphite and graphite by Raman spectroscopy, International Journal of Coal Geology 159 (2016), pp 48-56

10. Zheng, Z.; Zhang, J.; Huang, J. Y.: Observations of microstructure and reflectivity of coal graphites for two locations in China, International Journal of Coal Geology 30 (1996), pp 277-284

11. Kwiecińska, B.; Petersen, H.: Graphite, semi-graphite, natural coke and natural char classification - ICCP system, International Journal of Coal Geology 57 (2004), Nr. 2, pp 99-116

12. Wissler, M.: Graphite and carbon powders for electrochemical applications, Journal of Power Sources 156 (2006), Nr. 2, pp 142-150
13. Badenhorst, H.; Focke, W.: Comparative analysis of graphite oxidation behaviour based on microstructure, Journal of Nuclear Materials 442 (2013), 1-3, pp 75-82

14. Chaika, E. F.; Maryasev, I. G.; Platonov, A. A.: Graphite. Evaluation of quality for refractory industry, Refractories and Industrial Ceramics 57 (2017), Nr. 5, pp 449-461

15. Mitchell, C. J.: Industrial minerals laboratory manual: flake graphite: Technical Report WG/92/030, British Geological Survey

16. Mukhopadhyay, P.; Gupta, R. K.: Graphite, Graphene, and their Polymer Nanocomposites, Boca Raton: Taylor \& Francis Group, 2013

17. Simandl, G. J.; Kenan, W.M.: Microcystalline Graphite: Geological Fieldwork 1997, Paper 1998-1, British Columbia Geological Survey

18. Kraus, U. H.; Schmidt, H. W.: International Strategic Minerals Inventory Summary Report - Natural Graphite: U.S. Geological Survey Circular 930-H, 1988

19. Lämmerer, W.; Flachberger, H.; Stelzer, G.; Twrdy, G.: FFG Endbericht - Projekt „Innovative Grafite“, Leoben, 2015

20. Scogings, A.: Graphite Focus: Graphite: Where size matters, Australia's Paydirt 2015, March 2015, pp 78-79

21. Kim, B. G.; Choi, S. K.; Park, C. L.; Chung, H. S.; Jeon, H. S.: Inclusion of Gangue Mineral and its Mechanical Separation from Expanded Graphite, Particulate Science and Technology 21 (2003), Nr. 4, pp 341-351

22. Bulatovic, S. M.: Beneficiation of graphite ore, in: Bulatovic, S. M. (ed.): Handbook of flotation reagents: Chemistry, theory and practice; flotation of industrial minerals, Kidlington, England: Elsevier, 2015, pp 163-171

23. Salgado, M. R.: Upgrading graphite by flotation at Bogala Mines in Sri Lanka, Journal of Central South University of Technology 8 (2001), Nr. 3, pp 193-196

24. Nguyen, A. V.; Schulze, H. J.: Colloidal science of flotation, vol. 118, Surfactant science series, New York: Dekker, 2004

25. Ravichandran, V.: Studies on the effect of chemical reagents on beneficiation of natural graphite, Doctoral Thesis, Karaikudi, Alagappa University, Department of industrial chemistry, 2012

26. Vasumathi, N.; Kumar, V.; Nayak, B.; Rao, S.; Prabhakar, S.; Raju B.: Beneficiation of low grade graphite ore of eastern India by twostage grinding and flotation, Journal of Mining and Metallurgy A: Mining 50 (2014), Nr. 1, pp 9-17

27. Ravichandran, V.; Eswaraiahb, C.; Sakthivel, R.; Manisankar, P.: Characterization and selection of flotation reagents to improve the graphite ore beneficiation, Indian Journal of Ultra Chemistry 2012, Vol 8., pp 1-10

28. Pugh, R. J.: Non-ionic polyethylene oxide frothers in graphite flotation, Minerals Engineering 13 (2000), Nr. 2, pp 151-162

29. Haus, R.: Making the most of your graphite, $4^{\text {th }}$ Graphite \& Graphene Conference, Berlin, 2014

30. Tamashausky, A. V.: Graphite: A Multifunctional Additive for Paint and Coatings, http://asbury.com/pdf/CoatingsPaper.pdf (17.06.2017)

31. Lu, X.; Forssberg, E.: Preparation of high-purity and low-sulphur graphite from Woxna fine graphite concentrate by alkali roasting Minerals Engineering 15 (2002), Nr. 10, pp 755-757

32. Zaghib, K.; Song, X.; Guerfi, A.; Rioux, R.; Kinoshita, K.: Purification process of natural graphite as anode for Li-ion batteries: Chemical versus thermal, Journal of Power Sources 119-121 (2003), pp 8-15

33. Zhao, H.; Ren, J.; He, X.; Li, J.; Jiang, C.; Wan, C.: Purification and carbon-film-coating of natural graphite as anode materials for $\mathrm{Li}$ ion batteries, Electrochimica Acta 52 (2007), Nr. 19, pp 6006-6011

34. Kim, J.; Kim, B.: Chemical and low-expansion treatments for purifying natural graphite powder, Physicochemical Problems of Mineral Processing 41 (2007), pp 37-49

35. Wang, H.; Feng, Q.; Tang, X.; Liu, K.: Preparation of high-purity graphite from a fine microcrystalline graphite concentrate: Effect of alkali roasting pre-treatment and acid leaching process, Separation Science and Technology 51 (2016), Nr. 14, pp 2465-2472

36. Lämmerer, W.; Flachberger, H; Stelzer, G; Twrdy, G: 2. Zwischenbericht FFG - Projekt „Innovative Grafite“, Leoben, 2014

37. Xie, W.; Wang, Z.; Kuang, J.; Xu, H.; Yi, S.; Deng, Y.; Cao, T.; Guo, Z.: Fixed carbon content and reaction mechanism of natural microcrystalline graphite purified by hydrochloric acid and sodium fluoride, International Journal of Mineral Processing 155 (2016), pp 45-54 
38. Zhang, M.: Main methods for graphite purification, https://www. linkedin.com/pulse/main-methods-graphite-purification-maryzhang (17.06.2017)

39. Korthauer, R. (Hrsg.): Handbuch Lithium-lonen-Batterien, Frankfurt: Springer Vieweg, 2013

40. Yoshio, M.; Brodd, R. J.; Kozawa, A. (Hrsg.): Lithium-lon Batteries: Science and Technologies, New York, U.S.A.: Springer, 2009

41. Ketterer, B.; Bosch, U.; Öttinger, O.: Graphite Powder Processing: A Key Element for High Performance Lithium-Ion Batteries, DKG-AKK Herbsttagung, 2014

42. Wang, H.; Ikeda, T.; Fukuda, T.; Yoshio, M.: Effect of milling on the electrochemical performance of natural graphite as an anode material for lithium-ion battery, Journal of Power Sources 83 (1999), pp 141-147

43. Yoshio, M.; Wang, H.; Fukuda, K.; Umeno, T.; Abe, T.; Ogumi, Z.: Improvement of natural graphite as a lithium-ion battery anode material, from raw flake to carbon-coated sphere, Journal of Materials Chemistry 14 (2004), Nr. 11, p 1754
44. Seifert, S.; Thome, V.; Karlstetter, C.: Elektrodynamische Fragmentierung: Eine Technologie zur effektiven Aufbereitung von Abfallströmen, in: Kozmiensky-Thomé, K. J.; Goldmann, D. (Hrsg.): Recycling und Rohstoffe, Band 7, Nietwerder: TK-VIg, 2014, S. 431-438

45. Weh, A.; van der Wielen, K. P.: Gepulste Hochspannungstechnologie: Neue Wege in der Aufbereitung von primären und sekundären Rohstoffen, BVÖ Aufbereitungstechnisches Seminar 2015, Leoben, 2015

46. Böhm, A.; Flachberger, H.: Überblick über Methoden der Mahlbarkeitsprüfung, BHM Berg- und Hüttenmännische Monatshefte 151 (2006), Nr. 6, S. 223-232

47. Flachberger $\mathrm{H}_{\text {.; }}$ Mandelberger, T.: Trennmechanismen in elektrischen Feldern - eine Bestandsaufnahme, BHM Berg- und Hüttenmännische Monatshefte 154 (2009), Nr. 4, S. 129-135

48. Lämmerer, W.: Tanzanian Graphite Report, Interner Bericht, 2017

49. Lewandowski, W.: Elektroscheidung von Rohgrafiten, persönliche Mitteilung, 2016 